\title{
Dzyaloshinskii-Moriya Interaction and Anisotropy effects on the Entanglement of Heisenberg Model
}

\author{
M. Kargarian, ${ }^{1}$ R. Jafari, ${ }^{2}$ and A. Langari ${ }^{1}$ \\ ${ }^{1}$ Physics Department, Sharif University of Technology, Tehran 11155-9161, Iran \\ ${ }^{2}$ Institute for Advanced Studies in Basic Sciences, Zanjan 45195-1159, Iran
}

(Dated: November 1, 2018)

\begin{abstract}
In this paper the the effect of Dzyaloshinskii-Moriya interaction and anisotropy on the Entanglement of Heisenberg model has been studied. While the anisotropy suppress the entanglement due to favoring of the alignment of spins, the DM interaction restore the spoiled entanglement via the introduction of the quantum fluctuations. Thermodynamic limit of the model and emerging of nonanalytic behavior of the entanglement have also been probed. The singularities of the entanglement correspond to the critical boundary separating different phases of the model. The effect of gapped and gapless phases of the model on the features of the entanglement has also been discussed.
\end{abstract}

PACS numbers: 75.10. Pq, 03.67.Mn, 73.43.Nq

\section{INTRODUCTION}

In recent years, the notion of entanglement has received much attention in quantum information theory due to its notable features in developing the idea of quantum computers and other quantum information devices. Entanglement is a purely quantum correlation without classical counterpart ${ }^{1}$ and has been realized as a crucial resource to process and send information in novel ways such as quantum teleportation, supercoding and algorithms for quantum computations ${ }^{2}$. Concerning the correlation content of the entanglement, states of systems in condensed matter physics may deserve the investigation of entanglement as a unique measure of quantum correlations. The interest will be intensified when we consider the relation between entanglement and quantum phase transition where a drastic change in the ground state of the system occur ${ }^{3}$. This change will occur at zero temperature where all thermal fluctuations get frozen and only surviving quantum fluctuations drive the phase transition. In the past few years the subject of many activities were to investigate the role of entanglement in the vicinity of quantum critical point for different spin models $4,5,6,7,8,9,10,11,12,13$.

Spin models provide not only a test ground for above issue but also as a play-ground for implementation of may quantum information protocols $\frac{14,15}{15}$. Among them are Ising model in transverse field (ITF) and XXZ models. Despite of their simple Hamiltonian, low energy behavior of many systems can be captured through them. Ising model in transverse field has the benefit of exact solvability through mapping to the free fermion mode ${ }^{\underline{3}}$. Such solvability provide the possibility to test the behavior of entanglement and its scaling close to the critical point of the system and perform a finite size scaling as has been done in the seminal work of Osterloh and his coworkers 4 . The scenario is different in the XXZ model where the entanglement between the two nearest-neighbor sites develops a maximum at the isotropic point $(\Delta=1)$ without any singularity in its first derivative $\underline{16}^{16}$ which vanishes at the critical point $\Delta=1$. However, the block-block entanglement $\frac{17}{17}$ of the spin- $1 / 2$ XX model with three-spin and uniform long range interaction shows a logarithmic and algebraic dependence on the size of block for different phases. Logarithmic divergences of the entanglement entropy is a general feature of all one-dimension critical systems where the coefficient of the logarithm is just the central charge of the underlying critical theory 18 .

The scaling of entanglement close to the phase transition and its connection to the universality class of the model can be further investigated through employing the renormalization group. This method as we will see in the next sections provides a rather analytic framework for treating the phases of the model even for those that are beyond the exact solution. In this stream the scaling of the entanglement govern by the critical exponent of the model ${ }^{19,20}$. However, The renormalization of quantum states has also been introduced in terms of matrix product states 21 .

Both Ising and XXZ models can be supplemented with a magnetic term, the so called Dzyaloshinskii-Moriya (DM) interaction, arising from the spin-orbit coupling. Based on the symmetry aspects 22 , It can be derived microscopically as a linear correction to the standard superexchange mechanism ${ }^{23}$. The interaction has the form $\sum_{<i j>} \vec{D}_{i j} \cdot\left(\overrightarrow{S_{i}} \times \overrightarrow{S_{j}}\right)$ where the sum is over the pairs of spins. Some quantum antiferromagnetic (AF) systems are expected to be described by DM interaction with the underlying helical magnetic structures. Ising model with DM interaction was extensively studied ${ }^{24}$. DM interaction drive the quantum fluctuations resulting in a phase transition in the model. Critical point separates the antiferromagnetic and chiral phases. Derivative of the entanglement diverge at the critical point with the critical exponent of the model.

In this paper we address the behavior of the entanglement in the XXZ model with DM interaction. Including the DM interaction makes the phase diagram rich with a critical line instead a single point 25 . First we employ the quantum renormalization group to have a tractable 
problem. Afterwards, the entanglement between degrees of freedom is treated through the renormalization. We will see that derivative of the entanglement becomes singular at the phase boundary and its scaling correspond to the gapless and gapped phases of the model. The organization of the paper is as follows. In the next section we briefly introduce the renormalization group approach. In the Sec(III) we exemplifies the effect of anisotropy and DM interaction. Then in Sec(IV) we turn on to discuss the scaling of the entanglement, and the Sec(V) is devoted to the conclusions.

\section{QUANTUM RENORMALIZATION GROUP}

The Quantum renormalization group presents a tractable version of treating quantum systems at zero temperatures. Through the renormalization the original model Hamiltonian is replaced by an effective one in cost of renormalizing coupling constants. In this way the original Hilbert space truncated to an reduced Hilbert space including the effective degrees of freedom. Getting ride of extra degrees of freedom gives rises to the flow of the coupling constants in the parameter space of the model. The version we employ to kill the degrees of freedom is Kadanoff's block approach since it is well suited to perform analytical calculations in the lattice models and they are conceptually easy to be extended to the higher dimensions $26,27,28,29$. In the Kadanoff's method, the lattice is divided into blocks in which the Hamiltonian is exactly diagonalized. By selecting a number of low-lying eigenstates of the blocks the full Hamiltonian is projected onto these eigenstates giving the effective (renormalized) Hamiltonian.

The Hamiltonian of XXZ model with DM interaction in the $z$ direction on a periodic chain of $N$ sites is

$$
\begin{aligned}
H(J, \Delta)= & \frac{J}{4} \sum_{i}^{N}\left[\sigma_{i}^{x} \sigma_{i+1}^{x}+\sigma_{i}^{y} \sigma_{i+1}^{y}+\Delta \sigma_{i}^{z} \sigma_{i+1}^{z}\right. \\
& \left.+D\left(\sigma_{i}^{x} \sigma_{i+1}^{y}-\sigma_{i}^{y} \sigma_{i+1}^{x}\right)\right] \quad D, J, \Delta>0
\end{aligned}
$$

We divide the chain into blocks each containing three sites. The block Hamiltonian $\left(H_{B}=\sum h_{I}^{B}\right)$ of the three sites and its eigenstates and eigenvalues are given in Appendix A of reference $\frac{25}{2}$. However, we give only the degenerate ground states since we need for them for evaluation of entanglement and subsequent discussions, as follows:

$$
\begin{array}{r}
\left|\psi_{0}\right\rangle=\frac{1}{\sqrt{2 q(q+\Delta)\left(1+D^{2}\right)}}\left(2\left(D^{2}+1\right)|\downarrow \downarrow \uparrow\rangle\right. \\
\left.-(1-i D)(\Delta+q)|\downarrow \uparrow \downarrow\rangle-2\left[2 i D+\left(D^{2}-1\right)\right]|\uparrow \downarrow \downarrow\rangle\right), \\
\left|\psi_{0}^{\prime}\right\rangle=\frac{1}{\sqrt{2 q(q+\Delta)\left(1+D^{2}\right)}}\left(2\left(D^{2}+1\right)|\downarrow \uparrow \uparrow\rangle\right. \\
\left.\left.-(1-i D)(\Delta+q)|\uparrow \downarrow \uparrow\rangle-2\left[2 i D+\left(D^{2}-1\right)\right]|\uparrow \uparrow \downarrow\rangle\right)\right),
\end{array}
$$

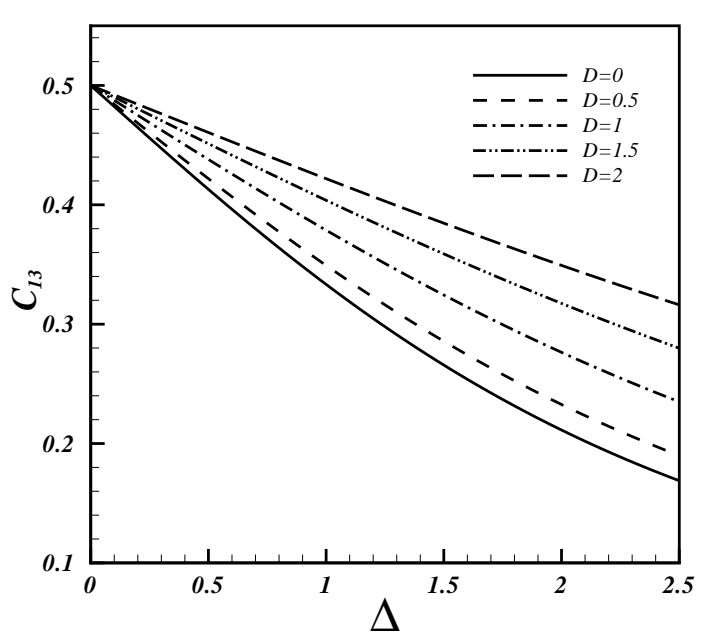

FIG. 1: Concurrence between first and third sites of a threesite model in terms of anisotropy for different values of DM interactions.

where $|\uparrow\rangle$ and $|\downarrow\rangle$ are eigenstates of the $\sigma^{z}$ Pauli operator and $q=\sqrt{\Delta^{2}+8\left(1+D^{2}\right)}$. The projection operator of the ground state subspace defined by $\left(P_{0}=|\Uparrow\rangle\left\langle\psi_{0}|+| \Downarrow\right.\right.$ \rangle$\left.\left\langle\psi_{0}^{\prime}\right|\right)$, Where $\left|\psi_{0}\right\rangle$ and $\left|\psi_{0}^{\prime}\right\rangle$ are the doubly degenerate ground states, $|\Uparrow\rangle$ and $|\Downarrow\rangle$ are the renamed base kets in the effective Hilbert space. We have kept two states $\left(\left|\psi_{0}\right\rangle\right.$ and $\left.\left|\psi_{0}^{\prime}\right\rangle\right)$ for each block to define the effective (new) site. Thus, the effective site can be considered as a spin $\frac{1}{2}$. The effective Hamiltonian is similar to the initial one, i.e,

$$
\begin{aligned}
H^{e f f}= & \frac{J^{\prime}}{4} \sum_{i}^{N}\left[\sigma_{i}^{x} \sigma_{i+1}^{x}+\sigma_{i}^{y} \sigma_{i+1}^{y}+\Delta^{\prime} \sigma_{i}^{z} \sigma_{i+1}^{z}\right. \\
& \left.+D^{\prime}\left(\sigma_{i}^{x} \sigma_{i+1}^{y}-\sigma_{i}^{y} \sigma_{i+1}^{x}\right)\right]
\end{aligned}
$$

where $J^{\prime}$ and $D^{\prime}$ are the renormalized coupling constants. The renormalized coupling constants are functions of the original ones which are given by the following equations.

$J^{\prime}=J\left(\frac{2}{q}\right)^{2}\left(1+D^{2}\right), \quad \Delta^{\prime}=\frac{\Delta}{1+D^{2}}\left(\frac{\Delta+q}{4}\right)^{2}, \quad D^{\prime}=D .(5)$

The above RG equations show that there is a phase boundary $\Delta_{c}=\sqrt{1+D^{2}}$ that separates the spin fluid phase, $\Delta<\sqrt{1+D^{2}}$, from the Néel phase, $\Delta>$ $\sqrt{1+D^{2}} \underline{25}$.

\section{ENTANGLEMENT ANALYSIS}

Many measures of entanglement have been introduced and analyzed $30,31,32,33$, but the most relevant to this work is the "entanglement of formation". For a reduced density matrix $\rho_{i j}$ of two qubits that arises after integrating 
out other degrees of freedom, the entanglement between two qubits is evaluated as $E=h\left(\frac{1}{2}+\frac{1}{2} \sqrt{1-C^{2}}\right)$, where $h$ is a binary entropy function $h(x)=-x \log _{2} x-(1-$ $x) \log _{2}(1-x)$ and $C$ denotes the concurrence ${ }^{31}$ defined as

$$
C=\operatorname{Max}\left\{\lambda_{1}-\lambda_{2}-\lambda_{3}-\lambda_{4}, 0\right\},
$$

where $\lambda_{k}(k=1,2,3,4)$ are the square roots of the eigenvalues in descending order of the operator $R_{i j}$ :

$$
R_{i j}=\rho_{i j} \tilde{\rho}_{i j} \quad, \quad \tilde{\rho}_{i j}=\left(\sigma_{1}^{y} \otimes \sigma_{2}^{y}\right) \rho_{i j}^{*}\left(\sigma_{1}^{y} \otimes \sigma_{2}^{y}\right) .
$$

In this section we consider only a three site block and study the effect of DM interaction and anisotropy parameter, i.e $D$ and $\Delta$, respectively, on the entanglement between two spins located on the sides of the block. To this end, let $\left|\psi_{0}\right\rangle$ be the ground state of the block. By tracing the density matrix $\rho=\left|\psi_{0}\right\rangle\left|\psi_{0}\right\rangle$ with respect to the middle site of the block, the obtained reduced density matrix and Eq.(6) give the expression for the concurrence in terms of couplings $D$ and $\Delta$.

For different values of DM interaction and anisotropy parameters, the plots of concurrence between first and third sites of the block $C_{13}$ have been depicted in Fig.(1). Consider first the case of $D=0$. In this case the model becomes the known $X X Z$ model. Large value of $\Delta$ implies the Neel state. Naturally this state is an product state without any entanglement between its constituents. As the anisotropy parameter reduces the quantum fluctuations arising from the transverse interactions have dominant effect and destroy Neel state. Indeed, the in-planar interactions drive the quantum correlations, i.e the qubits in the presence of quantum fluctuations are quantum correlated. The main message of the Fig.(1) is that the suppression of the entanglement can be compensated by tuning the DM interaction. In the absence of the anisotropy, the entanglement is insensitive to the DM value since both transverse interaction and DM term stimulate the quantum fluctuations. For nonzero value of anisotropy is clearly seen that the turning on the DM interaction restores the spoiled entanglement. The emerging of Neel state at large value of anisotropy and dominant quantum fluctuations at small value tempt to conclude that in the thermodynamic limit of the model there may occur quantum phase transition with the critical boundary depend on the competition between the parameters in the Hamiltonian. We will address this issue in the next section.

\section{THERMODYNAMIC LIMIT AND NON-ANALYTIC BEHAVIOR OF ENTANGLEMENT}

In this section we would like to see that how the quantum phase transition in the model, which can be signaled as unstable fixed point of RG equations, can be realized by examining the behavior of the entanglement. Indeed,

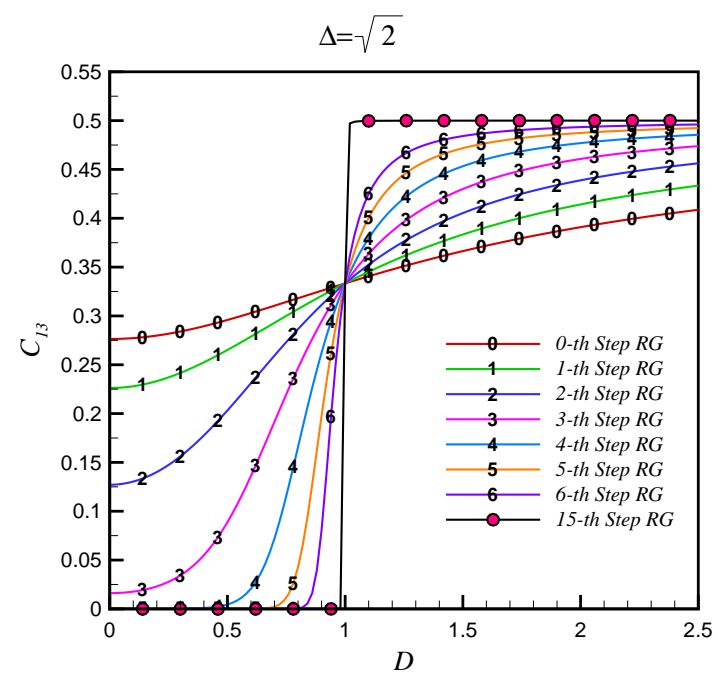

FIG. 2: (color online) Representation of the evolution of entanglement entropy in terms of RG iterations at fixed value of anisotropy $\Delta=\sqrt{2}$. Two different behavior of the entanglement at the large steps of RG correspond to the emerging phases of the model through the phase transition.

the non-analytic behavior in some physical quantity is a feature of second-order quantum phase transition. It is also accompanied by a scaling behavior since the correlation length diverges and there is no characteristic length scale in the system at the critical point. As we already pointed out the renormalization group allows us to capture the thermodynamic properties of the model by considering a block of a few sites that is analytically tractable. In fact the global properties of the model enter a few sites through the renormalizing of coupling constants. We exploit this advantage to study the scaling of the entanglement in the model. Notice that in the $n$ - th step of RG a system with size $n_{B}^{n+1}\left(n_{B}\right.$ is the number of sites in each block) describes effectively by a model consisting of only $n_{B}$ sites with the renormalized of coupling constants. The case of the XXZ model has been extensively studied 20 , where the critical point $\Delta=1$ separates spin-fluid and Neel phases. However, for the present model the contribution of the planar DM interaction tune the critical point of the model due to involving the quantum fluctuations. Since the DM interaction doesn't flow, as is clear from the RG equations in Eq.(5), it can be treated as a fixed parameter. Now we put the next step forward to see the evolution of the entanglement as the size of the system becomes large through the RG steps.

Zero-step RG represents a three-site model that its entanglement studied in the preceding section. However, the first-step RG stands for a nine-site model which effectively describes by a three-site model in the cost of renormalized coupling constants. In that case the entanglement measures the correlation between effective de- 


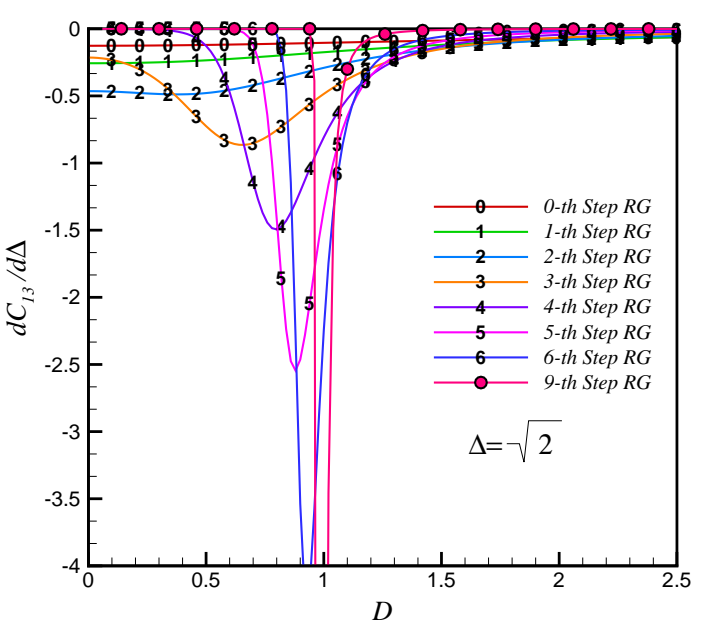

FIG. 3: (color online) First derivative of entanglement entropy and its manifestation towards divergence as the number of RG iterations increases (Fig 2).

grees of freedom. In each step of RG we can see the variation of the entanglement in terms of anisotropy parameter with the fixed value of DM interaction. All these data have been shown in Fig.(2). In this figure we have set the $\Delta=\sqrt{2}$. It reveals that as the thermodynamic limit is touched via the increasing of the RG steps, the entanglement develops two rather different features. Indeed, there is a value for $D=1$ that separates the different features. This value is exactly the critical point of the model which is consistent with $\Delta_{c}=\sqrt{1+D^{2}}$ if we set $\Delta=\sqrt{2}$. Different features of the entanglement correspond to the emerging phases on both sides of the critical point. For anisotropy parameter larger than the critical point the Neel ordering dominates the phase of the model while for anisotropy parameter less than the critical value the increasing of the planar quantum fluctuations spoil any magnetic ordering. This feature is not a specific character of the model arising at $\Delta=\sqrt{2}$. In fact for any value anisotropy $\Delta>1$ such behavior emerges with the only difference that the critical point is tuned into a new one.

Further insight on the non-analytic behavior can be probed through the diverging of the first derivative of the entanglement at the critical point as long as the the thermodynamic limit is approached. Plots related to the derivative of the entanglement at different RG steps have been shown in the Fig.(3). Each plot reveals a minimum which becomes singular as the critical point is touched. At the limit of large sizes of the model the singular behavior of the entanglement becomes more pronounced. One may wonder how such emerging singularity connects to the critical exponents or universality class of the model. To this purpose, we shall see that how position of minimum $D_{\text {min }}$ and minimum value itself $\left|\frac{d C}{d \Delta}\right|_{D_{\text {min }}}$ scale

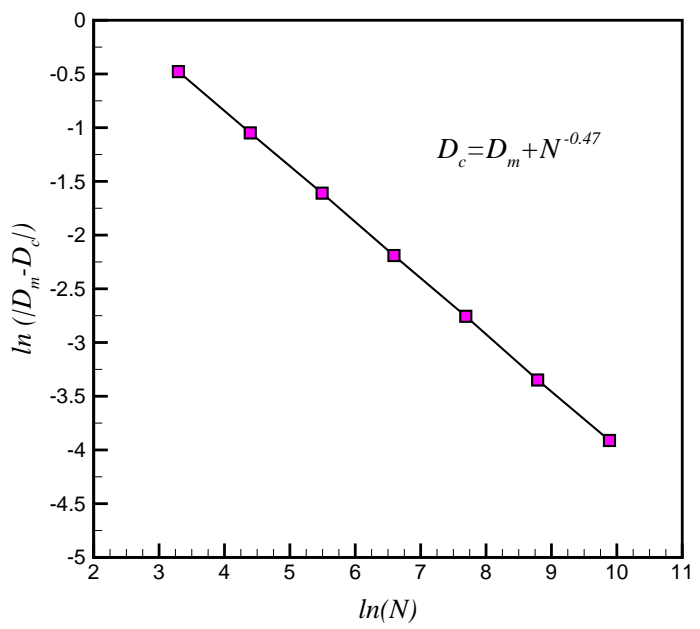

FIG. 4: (color online) The scaling behavior of $D_{m}$ in terms of system size $(N)$ where $D_{m}$ is the position of minimum in Fig. 3 .

with enlarging the size $N$ of the system. Such a computation determines the scaling law of entanglement in one-dimensional spin systems and explicitly uncovers an accurate correspondence with the critical properties of the model. The position of the minimum $\left(D_{m}\right)$ of $\frac{d C}{d \Delta}$ tends towards the critical point like $D_{m}=D_{c}-N^{-0.46}$ which has been plotted in Fig, Moreover, we have derived the scaling behavior of $y \equiv\left|\frac{d C}{d \Delta}\right|_{D_{m}}$ versus $N$. This has been plotted in Fig 5 which shows a linear behavior of $\ln (y)$ versus $\ln (N)$. The exponent for this behavior is $\left|\frac{d C}{d \Delta}\right|_{D_{m}} \sim N^{0.46}$. It should be emphasized that this exponent is directly related to the correlation length exponent, $\nu$, close to the critical point. It has been shown in Ref. 19] that $\left|\frac{d C}{d \Delta}\right|_{D_{c}} \sim N^{1 / \nu}$ and $D_{m}=D_{c}-N^{-1 / \nu}$.

Singular behavior of $\frac{d C}{d \Delta}$ corresponds to phase transition for any value of DM interaction. It exhibits a singular curve at the transition points. The latter can be characterized by analyzing the derivative of entanglement for all values of DM interaction. As an example, in Fig.(6) the derivative of entanglement in a three dimensional view has been shown. Noticeably, the divergencies in the derivative are in perfect correspondence with the parameter's value at which the phase transition occur. The crack in the figure is just the critical line separating antiferromagnetic from the spin fluid phases.

All above scaling functions hold for any value of anisotropy parameter as long as $\Delta>1$, which is a direct result of the fact that the parameter $D$ doesn't flow. This means that the emerging DM interaction term in the model doesn't change the universality class of the model. Thus far, taken the derivative of entanglement with respect to anisotropy parameter, the singularity appears at the critical point. To get more insight about the role of DM interaction in the singularity of the entanglement, 


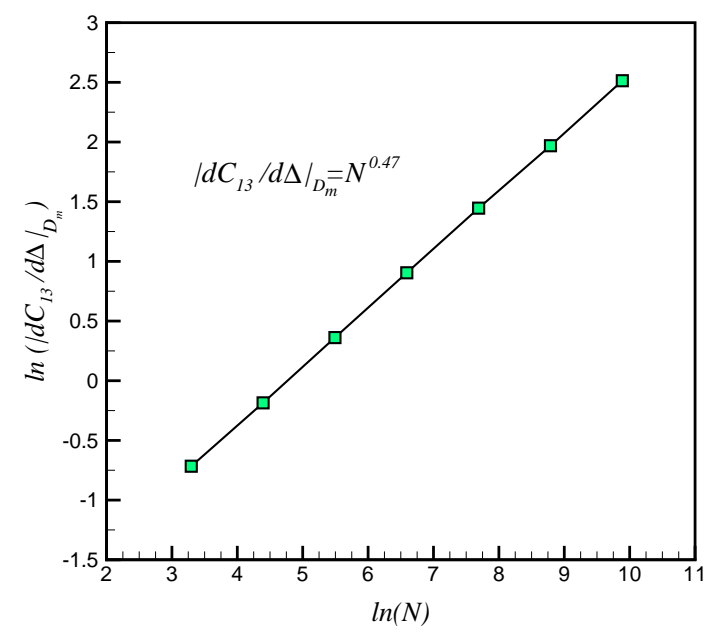

FIG. 5: (color online) The logarithm of the absolute value of minimum, $\ln \left(|d C / d \Delta|_{\text {min }}\right)$, versus the logarithm of chain size, $\ln (N)$, which is linear and shows a scaling behavior. Each point corresponds to the minimum value of a single plot of Fig[3]

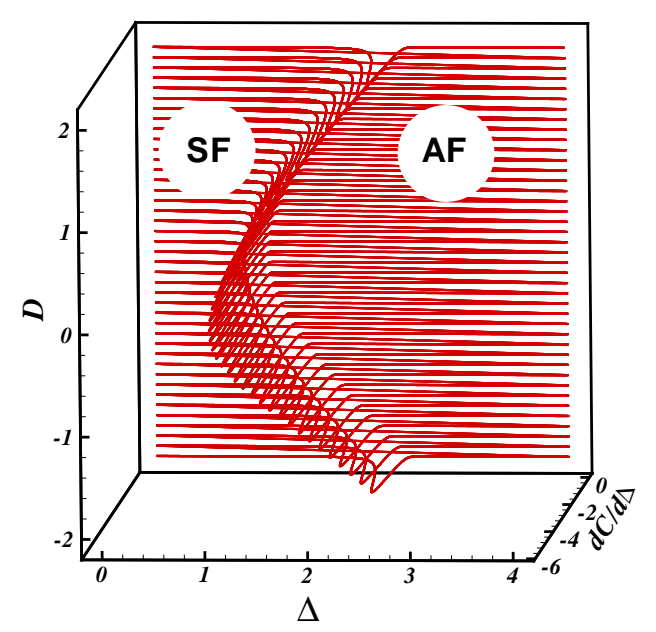

FIG. 6: The crack appearing in the derivative of the entanglement corresponds to the critical line of the model that separates antiferromagnetic (right) and spin fluid (left) phases.

it is convenient to plot the $\frac{d C}{d D}$ versus DM interaction as in Fig.(7). Even at the high steps of RG no singularity detect. Observe that the pair $(D=1, \Delta=\sqrt{2})$ stands for a point of singularity of derivative of entanglement with respect to $\Delta$ as in Fig.(31). However, there is no any signature of the divergence in latter quantity at this point when the derivative is taken with respect to $D$. This, again, verifies that the DM interaction have not any thing to do with the universality behavior of the

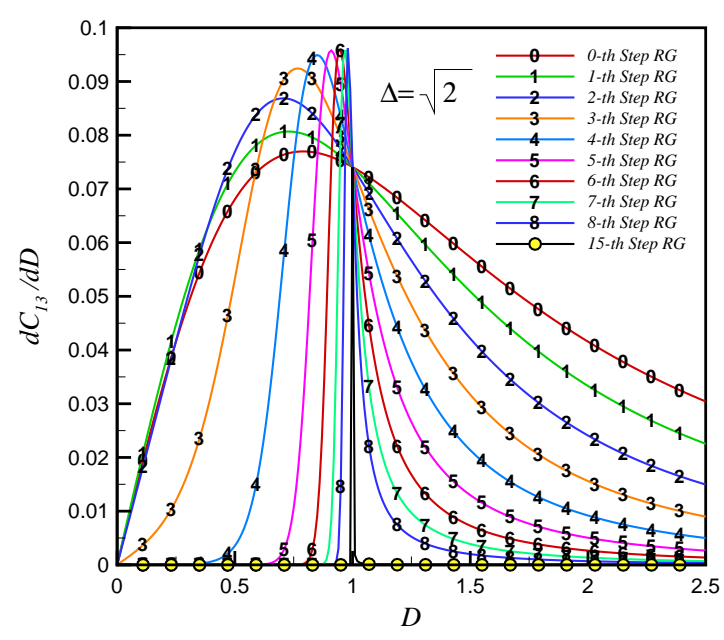

FIG. 7: The derivative of entanglement $\frac{d C}{d D}$ versus DM interaction for fixed value of anisotropy $\Delta=\sqrt{2}$. Even in limit of high steps of RG no singularity is observed.

model.

Indeed, for $\Delta>\sqrt{1+D^{2}}$ the long range behavior of the model fall into the the universality class of the Ising model that underlines the the appearance of the antiferromagnetic long range order 25 . We emphasize that here, the critical line $\Delta_{c}=\sqrt{1+D^{2}}$ from the Ising phase, i.e. $D_{m} \longrightarrow D_{c}^{-}$. This directly comes from the fact that the Ising phase is a gapped phase. Approaching the critical point, the gap is closed as $E_{g} \sim\left|D-D_{c}\right|^{\nu z}$, where $z$ is the dynamical exponent. Since in the limit of large sizes of the system the critical point is touched as $D_{c}-D_{m} \sim N^{-1 / \nu}$, we are left with the result that the gap of the Ising phase in the proximity of the critical point scales as $E_{g} \sim N^{-z}$. Whenever $\Delta<\sqrt{1+D^{2}}$, the model is gapless. This can be realized through a simple canonical transformation to the well known XXZ mode $l^{34,35}$ with the anisotropy $\tilde{\Delta}=\frac{\Delta}{\sqrt{1+D^{2}}}$. This implies that the model falls into a gapless spin fluid phase when $\tilde{\Delta}<1$.

Through this paper we have only considered the the entanglement between two sites living on sides of a threesite block, i.e. the middle site has bees traced out through the reduced density matrix. We would like to emphasize that we could already consider the entanglement between first two sites of the block. The entanglement between first and second sites of the block has been shown that in Fig.(8). At zero-step of RG that represent a threesite model, by increasing the DM interaction the entanglement between sites reduce. This is in correspondence with behavior in Fig.(2) where the entanglement between first and third sites is increased by increasing the DM interaction. This is expected since when two parties get more entangled, they restricts their entanglement with third party and vis versa that is a reminiscence of the 


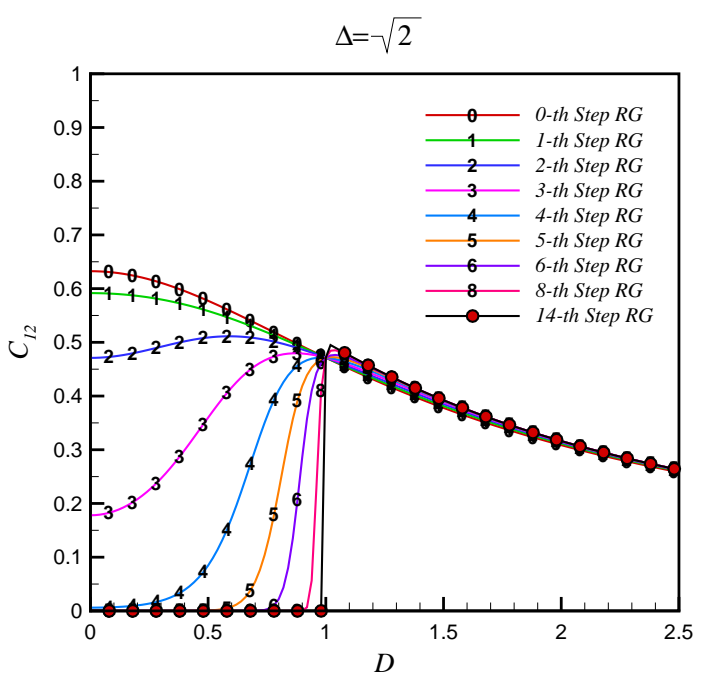

FIG. 8: The entanglement between first and second sites of the block in terms of DM interaction at different RG steps. As before the anisotropy parameter has been fixed at $\Delta=\sqrt{2}$.

monogamy property 36 of entangled objects. For $D<1$ that corresponds to the Ising phase, the entanglement between two sites is shaved out. RG equations runs the anisotropy to infinity dictating the spins to align. In contrary, for $D>1$ that corresponds to the gapless phase, all plots behave independent of RG steps. Observe that for $D<1$ neither first and second sites $\left(C_{12}\right)$ nor first and third ones $\left(C_{13}\right)$ in the large RG steps are entangled. This may not be surprising as in this limit the model is characterized by a polarized state. The situation is different for the gapless phase where the quantum fluctuations dominate the system suppressing the alignment of spins.

If we were to take the derivative of plots, again the singularity reveals itself at the critical point. Although $C_{13}$ and $C_{12}$ present different behavior, they share in exhibiting the critical behavior of the model.

\section{SUMMARY AND CONCLUSIONS}

Condensed matter systems have received impetus from the concepts developed in quantum information theory. The central to this is the entanglement being a unique measure of the quantum correlations. In this stream we studied the entanglement in a one-dimensional magnetic system in which many physical properties of realistic complex materials can be understood through it. This model is the well known XXZ model supplemented by a magnetic term arising from the spin-orbit coupling. The phase diagram of the model is determined by the anisotropy and Dzyaloshinskii-Moriya interaction (DM) parameters. In a simple model consisting of only three qubits, the increasing of anisotropy parameter favors the alignment of spins antiferromagnetically yielding a product ground state without entanglement. However, tuning the DM interaction tends to build an entangled state and restores the spoiled entanglement. This reviving of entanglement can be understood via the fact that the DM interaction contributes the strong planar quantum fluctuations that pose the alignment ordering.

The thermodynamic limit of the model realized through the renormalization group approach. The renormalization group not only allows us to derive the critical points as well as phase diagram of the model but via implementation of the RG steps we are able to keep track the variation of the entanglement as the size of the system becomes large. RG equations imply that the DM interaction tunes the critical point, i.e there is critical line instead a single critical point. However, the universality class of the model is unaffected in the presence of the DM interaction, which can be clearly seen from both RG equations and the scaling we obtained for the entanglement. The role of the DM interaction can be well understood by analyzing and comparing the derivative of entanglement with respect to DM interaction and anisotropy parameter. In the former case, even in the large RG steps, no singularity is observed. This can also be justified by mapping the model into the well known XXZ model using a canonical transformation. The derivative of the entanglement diverge at the all points of the critical line. The line singularity corresponds to the phase boundary separating the antiferromagnetic from the spin fluid phases. The singularity accompanies by some scaling functions with a emerging exponent that is related to the correlation length exponent close to the phase transition. We also verified that being gapped or gapless is relevant to the crossing the phase transition. Via the enlarging the size of the system, the singularity becomes more pronounced and touch the critical line from the gapped phase(antiferromagnetic). This phenomenon results the gap of the phase scales with the size of the system governed by the dynamical exponent.

\section{Acknowledgments}

This work was supported in part by the Center of Excellence in Complex Systems and Condensed Matter (www.cscm.ir).

\section{References}


tion and Quantum Communication (Cambridge University Press, Cambridge, 2000).

3 S. Sachdev, Quantum Phase Transitions (Cambridge University Press, Cambridge, 2000).

4 A. Osterloh, Luigi Amico, G. Falci and Rosario Fazio, Nature 416, 608 (2002).

${ }^{5}$ L.A. Wu, M. S. Sarandy, and D. A. Lidar,Phys. Rev. Lett 93250404 (2004).

${ }^{6}$ G. Vidal, J. I. Latorre, E. Rico, and A. Kitaev, Phys. Rev. Lett. 90, 227902 (2003).

7 J. Vidal, G. Palacios, and R. Mosseri, Phys. Rev. A 69, 022107 (2004).

8 T. J. Osborne and M. A. Nielsen, Phys. Rev. A 66, 032110 (2002).

9 I. Bose and E. Chattopadhyay, Phys. Rev. A 66, 062320 (2002).

10 F. Verstraete, M. Popp, and J. I. Cirac, Phys. Rev. Lett. 92, 027901 (2004).

11 L. Amico, R. Fazio, A. Osterloh and V. Vedral, Rev. Mod. Phys. 80, 517 (2008).

12 Shi-Jian Gu, Shu-Sa Deng, You-Quan Li, and Hai-Qing Lin,Phys. Rev. Lett. 93,086402 (2004).

13 Alberto Anfossi, Paolo Giorda, and Arianna Montorsi, Phys. Rev. B. 75, 165106 (2007).

14 D. Loss, D. P. Divincenzo, , Phys. Rev. A. 57, 120(1998).

15 R. Raussendorf and H. J. Briegel, Phys. Rev. Lett. 86, 5188 (2001).

16 Shi-Jian Gu, Hai-Qing Lin, and You-Quan Li, Phys. Rev. A 68, 042330 (2003).

17 P. Lou and J. Y. Lee, Phys. Rev. B 74, 134402 (2006).

18 P. Calabrese, J. Cardy, J. Stat. Mech. P06002 (2004).

19 M. Kargarian, R. Jafari, A. Langari, Phys. Rev. A 76,
060304(R) (2007)

20 M. Kargarian, R. Jafari, A. Langari, Phys. Rev. A 77, 032346 (2008).

21 F. Verstraete, J. I. Cirac, J. I. Latorre, E. Rico, M. M. Wolf, Phys. Rev. Lett. 94, 140601 (2005).

22 I. Dzyaloshinskii, J. Phys. Chem. Solids 4, 241 (1958).

23 T. Moriya, Phys. Rev 120, 91 (1960).

${ }^{24}$ R. Jafari, M. Kargarian, A. Langari, M. Siahatgar, Phys. Rev. B 78, 214414 (2008).

${ }^{25}$ R. Jafari, A. Langari, arXiv:0812.1862

26 M. A. Martin-Delgado and G. Sierra, Int. J. Mod, Phys. A 11, 3145 (1996).

27 G. Sierra and M. A. Martin Delgado, in Strongly Correlated Magnetic and Superconducting Systems, Lecture Notes in Physics Vo1. 478 (springer, Berlin, 1997).

28 A. Langari, Phys. Rev. B 58, 14467 (1998); 69, 100402(R) (2004).

29 R. Jafari, A. Langari, Phys. Rev. B 76, 014412 (2007); Physica A 364, 213 (2006).

30 Scott Hill and William K. Wootters, Phys. Rev. Lett. 78, 5022 (1997).

31 William K. Wootters, Phys. Rev. Lett. 80, 2245 (1998).

32 V. Vedral, M. B. Plenio, M. A. Rippin, and P. L. Knight, Phys. Rev. Lett. 78, 2275 (1997).

33 Michal Horodecki, Pawel Horodecki, and Ryszard Horodecki, Phys. Rev. Lett. 80, 5239 (1998).

${ }^{34}$ F. C. Alcaraz and W. F. Wreszinski, J. Stat. Phys. 58, 45 (1990).

35 D. N. Aristov, S. V. Maleyev, Phys. Rev. B 62, R751 (2000).

${ }^{36}$ V. Coffman, J. Kundu and W.K. Wootters Phys. Rev. A 61, $052306(2000)$ 